Cahiers $d u$ MONDE RUSSE

\section{Cahiers du monde russe}

Russie - Empire russe - Union soviétique et États indépendants

59/2-3 | 2018

Hors plan : L'économie informelle en URSS

\title{
La chose la plus monstrueuse était l'absence de couleurs
}

\section{Arsenij Roginskij}

Traducteur : Wladimir Berelowitch

\section{(2) OpenEdition}

\section{Journals}

\section{Édition électronique}

URL : https://journals.openedition.org/monderusse/10469

DOI : 10.4000/monderusse. 10469

ISSN : $1777-5388$

\section{Éditeur}

Éditions de l'EHESS

\section{Édition imprimée}

Date de publication : 1 avril 2018

Pagination : 369-390

ISBN : 978-2-7132-2746-2

ISSN : $1252-6576$

\section{Référence électronique}

Arsenij Roginskij, «La chose la plus monstrueuse était l'absence de couleurs », Cahiers du monde russe [En ligne], 59/2-3 | 2018, mis en ligne le 01 avril 2018, consulté le 08 janvier 2022. URL : http:// journals.openedition.org/monderusse/10469; DOI : https://doi.org/10.4000/monderusse.10469 


\section{LA CHOSE LA PLUS MONSTRUEUSE ÉTAIT L'ABSENCE DE COULEURS}

Aujourd'hui, nous sommes le 30 mars 2009, je viens d'avoir 63 ans ${ }^{1}$. Cela paraît un très grand âge, et je me rappelle toujours comment, lorsque j'étais gamin, je voyais mon professeur de 40 ans : il me semblait infiniment adulte. Non pas vieux, mais terriblement adulte. Quant aux sexagénaires, bien sûr, c'étaient pour moi des vieillards, mais chose étrange, aujourd'hui je ne me considère pas du tout comme un vieil homme, et même, parfois, je ne me sens pas tellement adulte. C'est comme s'il y avait déjà un très gros morceau de vie derrière moi, mais aussi comme si j'en avais encore trois fois plus devant moi qui m'attendait, même si, bien sûr, je sais que c'est une illusion. Donc je suis né le 30 mars 1946, et je suis né dans une certaine ville qui est totalement inconnue des Français et peu connue des Russes : la ville de Vel'sk, dans la région d'Arhangel'sk. Et toute ma vie, j'ai eu dans mon passeport ces mots : « la ville de Vel'sk » et, toute ma vie, j'ai éprouvé une sorte d'excitation en les lisant alors que je n'y suis jamais retourné, seulement, pour la première fois, il y a quelques années.

Mais si ce nom est pour moi important, c'est parce que, bien sûr, ce n'est pas la ville de Vel'sk qui fut mon lieu de naissance, mais l'infirmerie ${ }^{\circ} 4$ du camp de la Dvina septentrionale, qui faisait partie de la Direction principale des camps de construction de chemins de fer, en abréviation le Sevdvinlag du GULŽDS².

Mon père s'y trouvait alors. Mon père, Boris Roginskij, se prénommait en réalité Baer. C'est un nom juif qui, peu à peu et naturellement, s'est changé en « Boris » au cours des années trente, oui, dès les années trente. Il paraît même que dans son premier passeport, lorsque la population fut passeportisée en 1933,

1. Cet entretien a été recueilli en 2009 par Alain Blum, Sabine Breuillard et Marta Craveri dans le cadre du projet « Archives sonores : mémoires européennes du Goulag », coordonné par Alain Blum, Marta Craveri et Valérie Nivelon, http://museum.gulagmemories.eu. La version audio originale de cet entretien est accessible en ligne à l'adresse http://museum.gulagmemories.eu/fr/media/arseni-roginski

2. Severo-Dvinskij lager' Glavnogo upravlenija lagerej železnodorožnogo stroitel'stva. (NdT) 
c'était écrit « Boris », mais qu'il insista pour qu'on ajoute « Baer » entre parenthèses. Ce qui est assez important.

$\mathrm{Au}$ moment de ma naissance, mon père avait déjà purgé sa peine de camp. Il était ingénieur. Il était né comme tout un chacun dans la zone de résidence, dans un petit shtetl, Rečica, en Biélorussie. Dans une grande famille juive, pauvre, vraiment très pauvre. Dès la révolution, l'aîné partit faire ses études à Petrograd. C'était une grande libération pour les Juifs, cette possibilité de s'arracher au shtetl, d'aller faire des études. Et puis le reste de la famille suivit, d'abord les enfants, puis les parents. Mon père a fait des études au cours des années vingt, à Petrograd puis Leningrad, à l'Institut d'électrotechnique, et après avoir achevé ses études, il est devenu ingénieur à l'énorme usine Elektrosila, à Leningrad. En 1938, pendant la Grande Terreur et les arrestations de masse, mon père fut arrêté comme presque tous les ingénieurs de cette usine, qui furent tous accusés d'avoir conspiré contre le pouvoir soviétique, d'avoir eu des visées terroristes (assassiner Stalin et les autres, et ainsi de suite). Mais mon père fut arrêté assez tard, c'était en février 1938, et il s'est trouvé qu'il était encore en cours d'instruction quand l'ère de Ežov s'est terminée. Or Ežov, le commissaire du peuple à l'Intérieur, par qui, principalement, la terreur avait été administrée, fut démis de ses fonctions en novembre 1938, et remplacé par Lavrentij Berija. Berija commença par un réexamen des affaires instruites sous Ežov. On annonça que Ežov avait spécialement cherché à nuire, qu'il avait arrêté des innocents et au cours des mois qui suivirent, un petit contingent de personnes fut libéré, très petit bien sûr ; tous ces chiffres existent. Mais le plus important est ailleurs : un très grand nombre de dossiers de ceux qui se préparaient déjà à être condamnés à mort furent envoyés à une instance non judiciaire, le Conseil spécial près le NKVD. Or ce conseil n'avait pas le droit de condamner à mort, de sorte que début 39, il prononça seulement des peines légères, 5, 8, 10 ans de camp, et les gens furent envoyés en masse dans les camps à la seule fin de désemplir les prisons surpeuplées par Ežov. Et mon père eut une chance inouïe. S'il avait été arrêté trois mois avant, ils auraient eu le temps de le fusiller. S'il avait appartenu à la direction de l'usine — directeur ou directeur adjoint — il aurait été fusillé aussi, parce que cet organe judiciaire qu'était le Collège militaire de la Cour suprême continuait de fonctionner très activement à la fin de l'année 1938 et au début de l'année 39. Mais lui, en somme, il était ingénieur, à peine plus haut que le niveau moyen. Il était chef d'atelier. C'était un personnage important, mais ce n'était quand même pas du gibier pour le Collège militaire. Et il eut de la chance, il fut envoyé en camp dans le Nord, dans la région d'Arhangel'sk. Et se retrouva au camp de la Dvina septentrionale.

Les ingénieurs, là-bas... c'est important à comprendre pour tous ceux qui étudient l'histoire du Goulag : nos camps étaient quand même nécessairement chargés de faire quelque chose, ils n'étaient pas conçus comme des camps d'extermination, ce n'était pas leur fonction. Certains camps, à certaines époques, furent de facto des camps d'extermination, à cause de leur forte mortalité, par exemple celui de Karaganda en 1942-1943, où les effectifs des détenus pouvaient se renouveler entièrement trois fois par an, tant la mortalité était énorme. Ou encore les 
camps de la Kolyma en 1939-1943 : une mortalité démentielle. Mais quand même, tous ces camps avaient pour fonction principale des travaux, et celui de mon père devait construire des chemins de fer. Un chemin de fer qui était destiné à remplir une fonction stratégique, il menait à Vorkuta, qui est un bassin houiller, il fallait donc transporter du charbon. Le camp de la Dvina septentrionale était chargé du tronçon Konoša - Kotlas, ce sont deux petites villes. Les ingénieurs y valaient de l'or. Surtout ceux qui pouvaient être utilisés dans leur spécialité, et lui, apparemment, était vraiment un ingénieur-électricien très doué et en plus, comme on dit chez nous, il n'était pas manchot, il avait des mains en or, ce qui est très important dans les camps, parce qu'on manque toujours de matériel de remplacement et alors il faut inventer quelque chose. Rappelez-vous la célèbre nouvelle de Šalamov sur l'ingénieur Kipreev ${ }^{3}$ qui invente un procédé pour réparer des ampoules. C'est de la science-fiction! Des ingénieurs de ce genre, qui n'étaient pas seulement intelligents en théorie, mais aussi en pratique, valaient vraiment de l'or. C'est pourquoi mon père a eu de la chance : il n'est pas resté très longtemps, seulement six mois, aux travaux communs. Les travaux communs, ça voulait dire creuser la terre, faire des trous avec des barres à mine, poser les rails, poser les traverses, etc., etc., etc. Autrement dit, c'étaient des travaux très durs, qui menaient effectivement assez rapidement à un épuisement de l'organisme et par conséquent à la maladie, souvent à la mort.

Il a passé relativement peu de temps aux travaux communs, dès que les chefs du camp ont eu vent de la présence d'un ingénieur-électricien, il a été immédiatement affecté à cette fonction. Pratiquement, qu'est-ce que ça veut dire ? Ça veut dire que tant qu'il était détenu, il vivait dans le baraquement commun, mais que la partie du baraquement où étaient les ingénieurs était généralement séparée des autres, vous comprenez ? Et les ingénieurs vivaient dans des conditions légèrement meilleures. Ce que ça signifie concrètement ? Hum, là, c'est vraiment un sujet très difficile et j'ai mis des années de ma vie à le comprendre. Des conditions légèrement meilleures, ça veut dire que vous n'avez pas seulement droit à un simple châlit, mais aussi à un petit matelas, même s'il est rempli de n'importe quoi. Et puis ces ingénieurs, s'ils étaient appréciés des chefs, s'ils devenaient indispensables, ils ne recevaient pas seulement un petit matelas, mais pouvaient aussi avoir des morceaux de tissu qu'ils pouvaient utiliser comme des draps. C'est une chose très importante. Séparés des criminels de droit commun, ces ingénieurs risquaient moins d'être volés, on ne pouvait pas les dépouiller de tout ce qu'ils avaient, cela aussi est important. L'ingénieur pouvait garder son oreiller ! Au camp, l'oreiller est un des objets les plus précieux. C'est facile à comprendre : il fait froid, il y a du vent ; même si tu es ingénieur, il faut quand même se rendre au rassemblement du matin. Il faut quand même se mettre en colonne par cinq, rester là les mains derrière le dos, tête levée, crier je ne sais pas quoi quand on t'appelle. On est transi par le vent et

3. Dans les Récits de la Kolyma de Varlam Chalamov. Voir : V.T. Šalamov, « Žitie inženera Kipreeva [La vita de l'ingénieur Kipreev] », in Sobrenie sočinenij v četyrex tomax [Euvres en quatre tomes], t. 2, M. : Hudožestvennaja literatura, Vagrius, 1998, p. 150-163. (NdT) 
c'est pour ça que cette chose terrible, dont souffrent aujourd'hui des intellectuels en liberté comme vous et moi parce qu'ils passent leur temps devant un ordinateur sans tourner le cou, eh bien elle atteint tous les gens normaux, parce qu'ils attrapent froid au cou. Or comment dormir quand on a mal au cou ? L'oreiller, c'est l'objet qui accompagne toujours un voleur. Aujourd'hui encore, un vrai bandit ne circule pas de camp en camp sans son oreiller. On n'est pas forcé d'avoir une couverture : on peut arriver à en obtenir une de l'administration, on peut la prendre de force à un autre détenu. On peut même dormir sur une planche, mais il faut poser sa tête sur quelque chose de moelleux, pour ne pas avoir mal au cou. Mon père avait un oreiller. Et donc, d'un côté, c'était bien sûr un vrai camp, très dur, mais d'un autre côté, par rapport aux détenus ordinaires, sa position était, pour ainsi dire, relativement privilégiée. Et puis, bien évidemment, si vous êtes ingénieur, vous devez circuler, vérifier, mais comment ça peut se passer ? Mettons qu'à une dizaine de kilomètres d'ici, il y a je ne sais pas quoi, une machine électrique qui est en panne. Il faut la réparer. L’ingénieur prend un ouvrier spécialisé et il y va. Si vous comprenez le fonctionnement économique du Goulag, vous devez savoir, je suppose, pourquoi nos camps étaient si peu rentables, pourquoi le travail forcé, en règle générale, ne donnait pas les résultats qu'attendaient, que réclamaient aussi bien l'administration des camps que toutes ces hiérarchies supérieures. Ce n'est pas seulement parce qu'un homme qui travaille au bâton, de force, sous la contrainte, et qui n'est pas payé pour cela, qui est mal nourri, ce n'est pas parce que cet homme ne veut pas remplir les normes et ne les remplira pas, même s'il en est capable. Bien sûr que ce n'est pas seulement pour ça. Il y a encore mille raisons, et la raison principale, c'est le cumul entre le problème du régime pénitentiaire et celui de l'efficacité du travail. Les exigences qui relèvent du régime du camp viennent d'en haut, de Moscou. Tandis que le plan, lui, doit être réalisé ici, sur place. Selon les exigences du régime pénitentiaire, toute brigade qu'on a besoin d'envoyer à des travaux quelconques, même si elle ne comprend que cinq personnes, doit être, comme on dit, " escortisée », c'est-à-dire qu'elle doit être accompagnée par des gardiens, un, ou deux, ou vingt. Mais comment garder des prisonniers si on construit une voie ferrée ? Il y en a un qui va travailler à dix kilomètres du camp, un autre à vingt, encore un autre à trente. Le petit camp suivant qu'on va construire sera à cinquante ou soixante-dix kilomètres. Mais à des petites distances du camp de base, dix, quinze kilomètres, vous devez emmener les gens à pied. Il faut donc les « escortiser ». Alors que matériellement, il n'y a pas assez de gardiens pour ça. D'où une quantité invraisemblable de problèmes de toutes sortes. Y compris les problèmes économiques et c'est pourquoi aussi l'administration des camps s'efforçait de faire passer le plus possible de détenus en ... « désescortisés » comme on les appelait : on laissait à des détenus le droit de circuler sans escorte. Où voulez-vous qu'ils s'enfuient? Ils n'avaient pas d'endroit où aller, vous comprenez ? Tout le monde le comprenait très bien. Mais si tel ou tel détenu a été condamné au titre de l'article 58-6 ou 58-8, c'est-à-dire pour terrorisme, sabotage et ainsi de suite, on n'avait pas le droit de le « désescortiser ». Tandis que là, chance inouïe : mon père avait écopé l'article 58-10, à savoir « agitation et propagande antisoviétiques » — « contre-révolutionnaires » selon 
la terminologie de l'époque. Cet article-là pouvait être « désescortisé ». Dans des cas spéciaux, mais c'était quand même possible. Bien sûr, le cas de mon père était justement spécial. Donc, premièrement, le fait qu'il était ingénieur, deuxièmement, le fait qu'il avait le droit de se déplacer sans escorte. C'était important.

Bon, les années passent, c'est la guerre, mon père est en camp. Et ma mère pendant ce temps ? Ma mère, elle était aussi d'origine juive, mais à la différence de mon père, elle n'avait strictement rien à voir avec la culture nationale juive, ni avec quoi que ce soit de ce genre, jamais, parce que son père avait déjà été un journaliste russe bien avant la révolution, rédacteur d'un grand journal en Crimée, ami d'écrivains russes. C'était le milieu social de la culture russe, et ma mère en était issue. Ils vivaient à Simferopol, c'est en Crimée. Au cours des années vingt, ma mère s'installa d'abord à Moscou, puis à Leningrad, elle aussi pour faire des études, mais là il s'agissait de tout autre chose, elle fut prise au studio du Théâtre d'Art pour devenir actrice. Ensuite, elle se passionna terriblement pour l'étude du théâtre, pour la théorie, et elle combina les deux choses. Elle était donc une jeune actrice, assez douée, je crois, mais sur scène elle fut lectrice. C'était une époque où la récitation de poésie était très à la mode. Et ces récitals, quand l'acteur déclamait de la poésie et même de la prose, remplissaient de grandes salles entières. Puis cette époque a pris fin, au début des années trente, et elle est revenue à la fin des années cinquante. Moi-même, encore gamin, j'en ai été victime, je suis allé à ces concerts. Les poèmes de Blok, je ne les ai pas découverts dans des livres, je les ai entendus en salle. Cela a été très important, oui, très important pour moi. Et donc ma mère conjuguait, disons, cet amour pour le théâtre ou pour la récitation, et pour l'étude du théâtre, et elle a migré à Leningrad, où il y avait un institut d'art absolument étincelant, peut-être le meilleur de l'histoire de l'Union soviétique : l'Institut d'histoire de l'art, qui a eu aussi son histoire glorieuse et remarquable, mais passons. Toujours est-il qu'elle y acheva ses études, et elle est devenue à la fois actrice ou lectrice et spécialiste du théâtre, et c'est alors, vers 1930, qu'elle a fait la connaissance de mon père.

Où les jeunes gens doivent-ils traditionnellement faire connaissance à Leningrad ? Je veux dire les gens normaux et pas tous ces komsomols. À la salle de concert de la Philharmonie, bien sûr. Même maintenant, vous savez, aujourd'hui encore, une rencontre à la Philharmonie porte une certaine marque de qualité. Mais ça ne signifie pas du tout qu'à cette époque, la salle ait été fréquentée seulement par une élite. Non, il y avait là aussi des jeunes gens modernes, y compris ces, disons, révolutionnaires et même tout ce que vous voulez, toutes sortes de gens. Mon père, sur les opinions politiques duquel je reviendrai, y allait aussi, et ma mère aussi. $\mathrm{C}$ 'est là qu'ils se sont rencontrés. Ma mère m'a raconté l'histoire de cette rencontre une bonne dizaine de fois... et toujours pour me faire honte. En effet, j'ai passé ma vie à lire des livres et je ne sais rien faire de mes mains. J'ai des mains qui n'ont pas poussé là où il fallait - c'est une expression russe. Bref, elle me racontait qu'elle montait l'escalier de la Philharmonie avec ses nouveaux escarpins, quand soudain un de ses talons s'est cassé. Ce qui était une affreuse tragédie ! Car quand vous avez cassé un talon, que faites-vous ? Vous vous mettez à l'écart pour ne pas gêner les autres, et vous vous demandez s'il vaut mieux rentrer chez vous ou bien ôter 
le second escarpin et aller au concert déchaussée. Or il y avait là un grand jeune homme, il devait avoir alors 25 ans, qui marchait à côté d'elle ou la suivait de près. Et il lui dit : «Vous permettez? » Il s'approche d'elle, elle lui explique ce qui lui est arrivé, tout en tenant ses chaussures à la main. Alors il lui dit : «Attendez », il lui prend sa chaussure, sort un petit marteau de sa poche et lui répare son talon.

Ils ont eu... C'étaient des personnes très différentes. Lui, mon père, c'était un workaholic, un fanatique du travail. C'était une pléiade brillante, la première génération des ingénieurs soviétiques. Il faut savoir qu'il y a eu trois générations. La première avait fait ses études dans les années vingt, auprès des anciens ingénieurs et professeurs, ils avaient encore eu une bonne formation. En 1928, après le procès du Parti industriel, l'atmosphère changea complètement dans ce milieu des ingénieurs. Certains furent arrêtés, d'autres licenciés. D'autres encore partirent d'eux-mêmes pour être moins visibles, et ils furent remplacés d'abord par leurs élèves. Mais leurs élèves disparurent dans les déluges de 1937 : ces derniers, qui avaient fini leurs études en 1928 ou 29 furent arrêtés en grande masse sur l'accusation de sabotage et c'est une troisième génération qui prit le relais ; mais ceux-là étaient des avortons complets, vous comprenez, c'était déjà la troisième génération. Qui est sorti de nos instituts techniques à l'époque de la Grande Terreur? Leonid Brežnev, voilà, si vous voulez, un produit typique. Bien sûr il ne comprenait rien et ne pouvait rien comprendre à la technique. Il y avait là aussi des gens normaux, mais en fait le corps des ingénieurs se dégradait tout doucettement, de sorte qu'à la fin de l'époque soviétique, il est devenu quelque chose de pitoyable si on regarde les choses en face.

Mon père était ingénieur de $\mathrm{A}$ à $\mathrm{Z}$, et ma mère était d'une tout autre culture, elle était brillante, talentueuse. Et bien sûr, elle avait de la kacha dans la tête, parce que d'un côté elle aimait Blok, lisait et aimait dans son cœur Ahmatova tout en n'osant même pas se l'avouer, mais, d'un autre côté, elle aimait terriblement les poètes komsomols des années vingt, qui écrivaient des poèmes enflammés du genre de " Grenade, Grenade, ma Grenade » ${ }^{4}$, et elle a toujours gardé cette kacha d'art véritable et de cette poésie komsomole des années vingt. Elle n'a jamais eu de véritable vision du monde, pas même politique, mais elle était ainsi, une femme pleine de légèreté et de grâce.

Leur premier enfant, mon frère aîné, est né dès 1935. Le second, ma sœur aînée, en janvier 1938. Et deux semaines après, mon père fut arrêté. Et tout s'est brisé... Mon père en prison, aucune nouvelle de lui, personne ne sait rien, et elle a deux enfants sur les bras : que faire ? Nous sommes début 38. Et puis il arrive quelque chose d'assez intéressant : de façon instinctive, purement instinctive, elle entreprend de se sauver et de sauver ses enfants. C'est-à-dire qu'elle quitte l'appartement où elle a vécu, elle abandonne tout. Ce qui d'ailleurs va poser des problèmes au moment où elle réclamera un logement, après notre retour à Leningrad. Donc, elle laisse tout, elle disparaît, trouve des emplois, je ne sais quel travail de formation aux métiers du tourisme (tout en vivant avec ses enfants dans une base de

4. Du poète « prolétarien » Mihail Svetlov, paru en 1926 et devenu célèbre au point d'être mis plusieurs fois en musique. (NdT) 
tourisme près de Leningrad), correctrice dans un quelconque journal d'usine, bref elle change tout le temps d'emploi et se dit qu'elle pourrait être arrêtée à son tour et qu'alors, bien sûr, les enfants seraient expédiés dans un orphelinat et ainsi de suite. C'est ainsi qu'elle passe les années 38 et 39 : en fuyant.

J'ai eu une fois une conversation atroce avec ma mère, une conversation entre un historien et un sujet de l'histoire. L'une des erreurs les plus graves que nous autres, historiens, puissions commettre est de faire sentir à nos informateurs que nous en savons trop. Il ne faut jamais le faire, sous aucun prétexte. Or il y avait dans cette histoire une chose qui me préoccupait beaucoup et que je n'ai pu lui dissimuler. Il se trouve que j'ai été le premier, en 1991 ou 92 (c'était donc, déjà, à la nouvelle époque), à découvrir le fameux ordre 00486, celui qui prescrivait d'arrêter les épouses des condamnés. J'ai alors découvert beaucoup de ces ordres opérationnels que personne ne connaissait avant moi, mais je ne les ai pas publiés, j'en faisais des copies puis je les distribuais aux uns et aux autres, car il me semblait important que ça circule au plus vite. Par ailleurs, je suis conscient de mon perfectionnisme, qui m'a été inculqué par mes maîtres, et si j'avais préparé ces trois petits feuillets pour la publication, cela m'aurait pris un an. Parce que c'est un problème très intéressant et c'est ce qui fait que je n'ai presque rien publié jusqu'à présent, mais peu importe, d'autres gens l'ont fait et tant mieux, c'est une grande chance que tout ça se soit mis à circuler dans le monde. Or cet ordre commandait d'arrêter les femmes dont les maris avaient été condamnés à être fusillés, et seulement ceux-là, et uniquement lorsqu'ils avaient été condamnés à mort par le Collège militaire de la Cour suprême ou par des cours militaires : donc, seulement cette catégorie d'épouses. Mais papa avait été condamné par le Conseil spécial, de sorte que ma mère n'aurait pas été arrêtée, elle n'avait pas besoin de fuir, elle aurait pu rester dans son appartement. Je le lui ai dit et ce fut pour elle un coup terrible. Dans son for intérieur, elle était très fière d'avoir sauvé ses enfants de l'orphelinat et d'avoir évité l'arrestation. Et moi qui lui déballe : maman, on ne t'aurait rien fait. Ce fut un coup rude pour elle. Ce fut une de ces bêtises - j'en ai commis beaucoup dans ma vie, mais c'était une de celles que je me pardonne difficilement. J'aurais pu ne rien lui dire. Elle aurait très bien pu rester avec ce sentiment, disons, de fierté pour ce qu'elle avait fait.

Et puis c'est la guerre, et c'est Leningrad, et ma mère est prise dans le siège. Avec ses enfants. Or, c'est ce fameux premier hiver terrible qui a emporté les trois quarts du nombre total des victimes du siège. Et elle les a sauvés. Elle les a sauvés ! À ce moment-là, il y avait déjà ses parents à Leningrad, mes grands-parents. Ils ont brûlé toute la bibliothèque du grand-père, une énorme bibliothèque. Ma mère l'a fait en toute lucidité, vous savez. Elle a gardé quelques livres, bien sûr, je ne sais pas combien, peut-être 300, 500 ou même 1000, de la poésie. Tout le reste, elle l'a brûlé en toute lucidité. Très récemment, j'ai lu une nouvelle de Šalamov. Il s'agit d'une femme qui est la fille de Klimova (une révolutionnaire, morte de typhus pendant la guerre civile) et qui a fait du camp, où Šalamov l'a rencontrée. Et voici que cette femme, déjà sortie du camp, vient trouver à Moscou une dentiste qui a connu sa mère, et lui demande de l'aider à s'installer à Moscou. Šalamov l'accompagne. Et cette dentiste lui demande : « Avez-vous quelque chose qui ait de la valeur? ». Un 
tout autre langage ! Elle répond : «Seulement la médaille d'or de ma mère ». Et Šalamov lui chuchote à l'oreille : «Partons d'ici, partons d'ici ». Parce que c'était le dernier objet qui lui restait de sa mère - Klimova, une femme géniale, une sainte, révolutionnaire, terroriste, qui avait fait partie de l'Organisation de combat de Savinkov et qui s'était enfuie de la prison Novinskaja en 1910, etc., etc. ${ }^{5}$ " Vous devez sauver ça », lui dit Šalamov. « Je dois vivre » lui répond durement cette fille de révolutionnaire, elle sort un paquet de son sac et le donne à la dentiste. C'est ce qu'a vécu ma mère. Je peux imaginer le sentiment qu'elle avait éprouvé en expédiant ses livres dans le poêle. «Il faut vivre », elle n'avait pas de doutes. Elle trouva une place d'aide-soignante dans un hôpital, et recevait des produits alimentaires en rémunération de son travail. Elle réussit à emmener ses deux enfants en quittant Leningrad assiégée à la fin du printemps 42. Ils ont survécu tous les deux et Dieu merci, sont toujours en vie. Et puis, après un très, très long voyage, ils ont été évacués à Tachkent. Là, elle les a placés à l'orphelinat, a travaillé je ne sais où...

Et puis mon père fut libéré. Libéré, mais pas complètement. Vous vous rappelez que, à la suite d'une directive spéciale, tous les détenus qui finissaient de purger leurs peines pendant la guerre devaient être retenus dans les camps jusqu'à la fin des combats. Mais, en 1945, il y eut une nouvelle directive : ils devaient rester au camp tant qu'une décision spéciale ne serait pas prise à leur égard ; autrement dit ils étaient formellement libérés, mais en fait ils ne pouvaient quitter le camp. Et bien entendu, ils avaient besoin de mon père, absolument besoin, parce qu'il était ingénieur. Où en trouver un autre ? Donc il a changé de statut, mais est resté détenu. Quant à ma mère... C'est étonnant, vous savez, il y a des femmes qui écrivent des lettres à leurs maris tous les jours, et il y en a d'autres qui n'en écrivent jamais. Cela signifie-t-il pour autant qu'elles aiment moins leurs maris ? On n'en sait rien du tout, c'est un mystère de l'âme féminine. Voilà que je lis les lettres que mon père adressait à son frère depuis son camp (il avait un frère, un homme remarquable lui aussi, c'est une autre histoire à raconter ; il a été volontaire à la guerre, un savant très doué et intelligent, qui progressait très rapidement, il a soutenu des thèses) et je lis : « Sais-tu quelque chose à propos de Lena? Je n'ai rien reçu d'elle depuis huit mois. » Seigneur, j'imagine ce qu'il a dû ressentir. Lui est au camp et elle ne lui écrit pas pendant huit mois. Vous vous rendez compte?

Comment aiment les femmes : c'est encore un autre sujet. Mais maintenant, elle a senti qu'elle pourrait le voir ! Le voir ! Le toucher ! Elle attrape ses deux enfants, quitte Tachkent, traverse tout le pays, vers le Nord, vers le camp, vers lui, et elle $\mathrm{y}$ arrive, avec ses enfants. Le commandant du camp l'autorise à s'installer dans la «zone industrielle ». Il faut savoir que chaque camp est divisé en deux zones, la zone de résidence et la zone de travail ou « industrielle ». Pour passer de l'une

5. Il s'agit de Natal'ja Sergeevna Klimova (1885-1918) qui fut en effet terroriste dans l'Organisation de combat du Parti socialiste-révolutionnaire, plus tard compagne de Boris Savinkov. Elle participa à la célèbre évasion de 13 détenues de la prison pour femmes de Moscou Novinskaja en 1909 ; elle mourut à Paris de grippe espagnole, et non de typhus. Sa fille fit dix années de camp en 1937-1947. La nouvelle de Salamov, « Zolotaja medal' [La médaille d'or] », est parue dans Pod'em, 2, 1990, p. 156-185. (NdT) 
à l'autre, les détenus subissent la fouille, le contrôle, etc., dans les deux sens, ce qui n'empêche pas cette zone, bien sûr, d'être isolée du monde extérieur par des barbelés, une sentinelle : le camp forme un ensemble, juste divisé en deux zones. Et donc le commandant leur permet de planter une maisonnette (une seule pièce) dans la zone de travail, et ma mère avec ses enfants et, de façon semi-légale, mon père aussi sont autorisés à y vivre. Et à 1000 mètres de là, il y avait l'hôpital du camp ou l'infirmerie, et c'est là, il y a 63 ans, que je suis né [rire]. Et voilà !

Il faut savoir que dans cette petite ville de Vel'sk, non loin de laquelle se trouvait ce camp, il y avait aussi un hôpital municipal, où travaillaient des médecins libres. Je demande à ma mère : « Maman, pourquoi donc es-tu allée accoucher à l'hôpital du camp ? » Et elle : «Tu es fou ! Nous avions des médecins d'un tout autre niveau ! » Je suis donc né à l'hôpital du camp, alors qu'elle avait le droit d'aller à l'hôpital municipal. Intéressant, n'est-ce pas ? Et voilà que des années et des années passent et que je me retrouve soudain dans cette ville de Vel'sk. Je suis reçu comme un personnage important, accueilli par la responsable culturelle de la ville ou de l'arrondissement, une dame très gentille, d'à peu près mon âge. Nous roulons en voiture, et je lui dis : « Vous savez, je suis d'ici, moi. » Elle dit : «C'est vrai ? Ah, mon Dieu ». La conversation s'engage un peu et je lui dis : « J'aimerais voir l'endroit où je suis né. Il n'y a plus rien à la place du camp ? » Elle répond : « Non. Encore récemment il y avait le bâtiment de cette infirmerie très remarquable, de cet hôpital. Mais maintenant il ne reste rien, malheureusement. En fait, j'y suis née aussi, moi. » "Seigneur, lui dis-je, comment ça vous y êtes née ? Votre père était donc un détenu ? « Eh non! Mon père est le capitaine Untel. » Or son nom, je le connais ! C'était un des officiers du camp dont le nom est resté dans ma mémoire. Et dans notre mémoire familiale, vous comprenez. Donc cet officier préféra envoyer aussi sa femme accoucher à l'hôpital pour détenus. C'est un sujet assez fondamental.

Comme je vous l'ai dit, pendant tout le temps que j'ai porté dans ma poche mon passeport soviétique où figurait la ville de Vel'sk, je comprenais qu'il y avait là quelque chose de très particulier. C'était une sorte de mythe. Seulement, on m'avait emmené de là quand j'avais six mois. Le camp a été démantelé, liquidé. Et bien sûr, j'ai toujours rêvé d'y aller et j'ai toujours eu peur d'y aller. Quand je pense au passé, il m'est beaucoup plus facile d'être historien qu'homme, que témoin, même indirect, des événements. Je n'ai jamais pu, jusqu'à présent, lire le dossier d'instruction de mon père. Je ne pouvais pas. J'en ai lu pourtant des milliers ! Mais celui de mon père... je ne peux pas l'ouvrir, je ne peux pas.

Et puis il y a eu l'automne et l'hiver 1982, une période pour moi pénible. Il s'est trouvé qu'on me fit partir de mon camp parce qu'il y avait eu quelque chose comme une rébellion des détenus et que l'administration du camp avait décidé (vrai ou faux, c'est une autre histoire) que j'en avais été une des causes principales. Toujours est-il qu'à 11 heures du soir, toute une foule de gens fait irruption dans le baraquement, alors que j'étais déjà couché : « Roginskij, prenez vos affaires, dehors ». Comment ça, « avec vos affaires »? À 11 heures du soir, on ne convoque personne avec ses affaires ! Tout ça en pleine taïga, dans la république 
des Komis. Mon sac n'est pas prêt, rien n'est prêt. Aussitôt, je commence à y fourrer je ne sais quoi. Tout le baraquement est sens dessus dessous. Qui me donne des cigarettes, qui des allumettes, qui du sucre, et au final je me retrouve avec un sac gros comme ça! Je fais mes adieux avec mes amis, sors dans la nuit avec cet énorme fourre-tout que, de mon temps, on appelait un sidor. Quand on sort du baraquement, on se trouve sur la place d'appel où on doit se mettre en rang deux fois par jour, matin et soir, pour être comptés. Donc je traverse la place d'appel avec mon sidor monstrueux, trois gardiens devant, trois gardiens derrière. Vraiment, une situation romantique, vous savez! Je me serais pris pour un personnage littéraire. Du reste, le goulag est une immense expérience culturelle ou littéraire pour qui sait déjà beaucoup de choses à son sujet. C'est une sorte de complément personnel à la littérature des Mémoires, à Solženicyn, à Šalamov et aux autres. Donc, je marche et je me dis : «Mon Dieu, comme c'est beau, cette nuit, cette taïga, le sidor sur le dos, le camion, le berger allemand à l'arrière... ». On nous place à l'arrière : moi, un petit soldat et le chien. Puis ils font encore sortir deux gamins et on nous emmène tous à la prison de transit de la direction régionale $\mathrm{du}$ camp. Ils veulent liquider cette pseudo-révolte, c'est pourquoi il faut nous expédier d'urgence. Seulement personne ne peut nous envoyer au-delà de la prison de transit, parce que pour cela il faut un ordre venu d'en haut. Ni le chef du camp, ni celui de la direction générale des camps d'Ust'-Vym'-Lag, parce que je suis un détenu de régime commun et qu'il y a un seul camp de régime commun pour tout cet énorme ensemble, c'est celui où j'étais. Et donc, tant que Moscou ne dit rien, on ne peut m'envoyer nulle part. On m'enferme dans la prison et j'y reste longtemps, jusqu'à ce que mon sort soit réglé quelque part. Pourtant, les gens de ma direction essayent de se mettre d'accord avec ceux de la direction voisine. « Nous en avons un, ici, embarquez-le. » Alors on me met dans un train, parce que la voie ferrée, comme toujours dans les camps, passait tout près de la prison, pour qu'on puisse aller à pied depuis le wagon Stolypin jusqu'à la prison de transit. Voilà donc qu'on me fait faire du train. J'arrive dans un camp, ils ouvrent mon dossier, non, il y a quelque chose qui ne va pas, ils le referment et refusent de me prendre, et ça recommence. Deux mois ainsi. Ma famille a perdu ma trace, c'est la panique, qu'est-ce qui m'est arrivé, aucune lettre de moi, parce qu'il n'est pas question de courrier quand on est en transit, ce serait du jamais vu. Et puis un jour, on roule, on me balade un peu partout, on passe de la république des Komis à la région d'Arhangel'sk, et voilà qu'on me dit tout à coup : « Bon ! On se prépare, ça va être Vel'sk. » Et moi, en entendant ce nom, je me dis : « Seigneur, on va me débarquer à Vel'sk. » Il faut dire que le camp de Vel'sk était un camp de régime spécial. «On va me débarquer à Vel'sk, je vais sortir, marcher sur les pierres qui ont été foulées autrefois par mon père et je vais arriver à la zone, peut-être à la même zone que la sienne. Et la boucle sera bouclée. » Mais je fus emmené plus loin [rire]. Oui, malheureusement !

Ce camp fut démantelé à l'automne 1946. Les ingénieurs du camp, c'était fini. Donc problème pour mes parents : où aller, car ils étaient libres désormais ? C'est une question très importante pour un zek intelligent et expérimenté : où aller quand il 
a purgé sa peine ? Que faisaient les plus intelligents? Ils s'en allaient loin en Sibérie et y trouvaient du travail. C'est ce que firent des amis de mon père, ses compagnons de détention - le dernier d'entre eux est mort il y a quelques années —, ils sont partis à Noril'sk, ont travaillé au combinat de Noril'sk comme ingénieurs libres, sont rentrés à Moscou après le $\mathrm{XX}^{\mathrm{e}}$ congrès, après avoir gagné dix années de salaire avec les primes de grand froid en sus. Ce qui leur a permis de s'acheter des appartements coopératifs, ça venait alors seulement de commencer. Mais mon père se conduisit de façon extrêmement déraisonnable. C'était quand même un fils à maman bien juif, il aimait beaucoup sa petite maman, or la petite maman vivait à Leningrad, il avait donc très envie de revenir à Leningrad. Bien sûr, personne ne l'aurait embauché à Leningrad, mais à $300 \mathrm{~km}$ de la ville on construisait alors le barrage sur la Svir'. Autrefois il y avait eu là le camp de Svir'lag, mais il fut fermé au milieu des années trente, il restait des petits bouts de camps, on construisait le barrage de Svir'-2 ou Svir'stroj, où il fut, bien sûr, invité volontiers comme ingénieur-électricien. C'était seulement à $300 \mathrm{~km}$ de Leningrad, soit à quelques heures de train. Et donc, après avoir voyagé près d'un an dans l'Oural, dans certaines villes de l'Oural, nous nous fixâmes enfin là-bas. Cette localité portait le nom de Podporož'e, la ville de Podporož e dans la région de Leningrad. Là, mon père commença peu à peu à oublier le camp, et moi, je garde quelques vagues souvenirs de cette époque, car je commençais à grandir, quand, en janvier 1951, il fut à nouveau arrêté comme povtornik [récidiviste].

Il faut dire qu'en octobre 1947, le camarade Stalin eut à affronter un problème presque insoluble : les détenus qui avaient été condamnés en 1937 à dix années de camp étaient en train de terminer leur peine, il fallait donc en faire quelque chose. Et ils étaient nombreux, disséminés dans le pays dans toutes sortes de camps. Évidemment, il ne savait peut-être même pas combien d'entre eux avaient survécu, combien étaient morts, ces détails, bien sûr, ne l'intéressaient pas. Mais on était fin 47. Vous comprenez ce que ça signifie : c'était l'étape la plus marquante, la plus haute en couleur si j'ose dire, de ce que vous et moi appelons par convention « la Guerre froide ». Voilà encore un autre sujet : le goulag et la Guerre froide. Bref, on émit une ordonnance spéciale, selon laquelle on devrait arrêter de nouveau, mais sans nouvelles accusations, tous ceux qui avaient déjà été condamnés en vertu de l'article 58 du code pénal de la RSFSR et des codes des autres républiques soviétiques. Non, quand même pas tous ces gens, bien sûr, mais ceux dont l'acte d'accusation contenait des formulations ayant un rapport avec le terrorisme, la trahison de la patrie, l'espionnage, le sabotage. Et aussi ceux qui n'étaient pas dans ce cas, ou qui avaient été seulement condamnés en vertu de l'article 58-10, mais dont les verdicts avaient contenu des formulations sur leur appartenance aux trotskistes, boukhariniens, chliapnikoviens ou toute une série de catégories de ce genre. Tous ces gens, il fut prescrit non pas de les fusiller, oh non, mais de les exiler à perpétuité dans la région de Krasnojarsk. Relégation à vie dans la région de Krasnojarsk. Et la campagne démarra. La statistique de cette campagne demanderait un autre exposé, en quelque sorte scientifique. Les années les plus intéressantes furent bien sûr 1948 et 1949 , quand la campagne fut intense, puis elle se ralentit. Mon père fut arrêté en 
51. Pourquoi ? C'est facile à comprendre. Tant que le barrage était en chantier, on avait besoin de lui, et ce scélérat de chef le couvrait. Mais quand les travaux furent à peu près terminés, le chef cessa de le couvrir. Il fut d'ailleurs remplacé. Mon père fut donc arrêté et emmené à Leningrad. À Leningrad.

Ce qui suit n'est pas pour publication. C'est la longue histoire qui fait partie de ma vie : comment j'ai essayé de trouver les traces de ce qui était arrivé à mon père $^{6}$. En effet, ma mère alla plusieurs fois à Leningrad pour transmettre des colis à mon père, mais très vite, on lui dit qu'il n'était plus de ce monde : retournez à Podporož'e, - ce bourg ouvrier où elle vivait toujours et qui est devenu une ville, un chef-lieu de district dans la région de Leningrad - allez au bureau d'état civil, on vous donnera un certificat. Elle l'a fait, et on lui a donné un certificat, selon lequel il était mort d'une crise cardiaque à Podporož'e. Même la date indiquée était fausse : elle était antérieure à celle de son arrestation. Un certificat de pure forme, donc, et il était clair qu'il était mensonger. Mais en revanche, il était clair aussi qu'il n'était plus de ce monde.

Durant de longues années je cherchai à savoir. Sans succès. Dans les années soixante-dix, grâce à des tierces personnes, je réussis à entrer en contact avec un responsable du parti de rang moyen (parce que ce n'est pas le genre de personnes que je connaissais). Je lui dis : «Aidez-moi, aidez-moi s'il vous plaît à savoir ce qui s'est passé. » « D'accord », répond-il. Au bout de quelque temps, il me fait venir et me dit : «Tu ne dois pas chercher à savoir, et ne pose plus de questions. »C'est tout, nous ne savons toujours rien.

Quelques années après la mort de Stalin, en 55 ou 56, ce qu'on appelle chez nous l'époque de la « Réhabilitance précoce », un homme téléphone à notre oncle (au frère de mon père) et lui raconte la version suivante : il avait été compagnon de cellule de mon père, un jour mon père fut emmené à un interrogatoire et il n'en est pas revenu. Puis des gardiens sont venus prendre ses vêtements ; selon une légende qu'un des gardiens aurait prétendument racontée à cet homme, mon père aurait frappé l'enquêteur ou lui aurait jeté l'encrier à la tête et l'autre l'aurait abattu en plein interrogatoire. Voilà donc une autre version. La même version m'est parvenue d'une autre source, d'un autre homme qui avait été détenu avec mon père à la prison des Croix (Kresty).

Puis, au début des années quatre-vingt-dix, j'ai écrit une requête et reçu la réponse officielle que voici : l'instruction de l'affaire de mon père avait été achevée, il était accusé d'agitation et de propagande antisoviétique - OK, c'était la confirmation de l'accusation de 1938 — il avait été condamné à telle date, qui était même indiquée dans la réponse que j'ai toujours, il fut condamné à la relégation à perpétuité dans la région de Krasnojarsk et trois jours après ce qui découle de la chronologie des faits, il s'est pendu dans sa cellule...

6. Lors de l'entretien, Arsenij Roginskij n'avait pas souhaité que ce passage relatif à la fin de son père soit publié, or il en parle tout à fait librement dans l'entretien précédent «Arsenij Roginskij : la vérité et le droit». La rédaction a donc considéré possible la publication de ce passage dans la présente publication. 
J'ai envoyé mon frère aîné lire le dossier de mon père. C'est un homme intelligent mon frère aîné, il est journaliste, il a lu tout le dossier, sans prendre de notes, bien sûr, et il m'a dit : « Il y a là-dedans le procès-verbal de l'examen du corps, eh bien voilà, il paraît qu'il se serait pendu au radiateur de sa cellule, donc tout concorde. »

Et moi, je vous l'ai dit, je suis incapable de lire ces dossiers, ni le premier, ni le second, je ne le peux pas. Mais comprenez-le, c'est très difficile à croire. Il était père de trois enfants, juif, et un juif qui avait eu une éducation juive, ce n'est pas très caractéristique des juifs de se suicider, mais surtout, ce verdict de relégation perpétuelle, cela signifie quoi ? Ça veut dire qu'on vous embarque dans un wagon pour un mois - un mois et demi de voyage, puis qu'on vous débarque à une gare dans la région de Krasnojarsk. Mais mon père n'était pas un spécialiste de littérature ou un historien russisant, c'était un ingénieur qui n'était pas manchot, vous comprenez il aurait survécu n'importe où, il avait fait du camp, il avait trois enfants, et j'étais encore petit à l'époque, et il pouvait être sûr qu'il ne se serait pas passé trois jours avant que ma mère prenne le train et aille le retrouver. Donc ce n'est pas du tout vraisemblable, mais c'est la version officielle, et les documents sont faits de telle façon que nous ne pouvons rien dire... il n'y a pas d'autre version, sauf ces ouï-dire. Certes, d'après les récits de ma mère, il lui avait dit qu'il ne supporterait pas qu'on l'embarque une seconde fois. Mais que signifie « je ne le supporterai pas »? Une telle formulation pouvait aussi bien signifier qu'il ne supporterait pas de nouvelles tortures, parce qu'il se rappelait celles de 1937-1938. Seulement il ne fut pas torturé en 51, les « récidivistes » ne l'étaient jamais, c'était inutile parce qu'on n'attendait d'eux aucun nouvel aveu. On demandait seulement : « Pourquoi avez-vous été arrêté la première fois ? », le chef d'accusation était repris et c'était tout.

Voilà donc comment finit l'histoire de mon père.

Quant à ma mère, elle avait acquis une nouvelle profession grâce au camp, parce que, revenons à cette période, quand elle était allée retrouver mon père, elle était trop énergique pour ne rien faire. Elle organisa immédiatement un théâtre du camp et monta des spectacles. Ses souvenirs du camp, ce sont des souvenirs des spectacles qu'elle a montés : qui a joué, comment, s'il était bon ou mauvais et qui était très doué : « il y avait Untel, un bandit terrible, il était doué, mais doué, c'est à peine croyable. Un jeune premier classique. Je n'en ai plus jamais vu de pareil. » Par la suite, elle a toute sa vie dirigé des troupes de théâtre amateur. Dans ce bourg de Podporož'e, où nous avions vécu, elle l'a fait aussi. Naturellement, cela devint impossible après l'arrestation de mon père, et pendant plusieurs années elle a été institutrice. Bien sûr, cela aussi était interdit, mais on avait besoin d'une enseignante d'allemand. Or elle savait l'allemand, comme ça, simplement parce que dans son enfance elle avait eu une bonne allemande, et elle a enseigné l'allemand. Avant cela, elle avait eu un autre emploi, il fallait bien qu'elle nous nourrisse. Et puis elle a travaillé jusqu'à sa retraite comme éducatrice dans un extraordinaire home d'enfants musical et en même temps elle a dirigé des spectacles et ensuite, à la retraite, elle a vendu des brochures dans un kiosque, et elle était heureuse. 
Elle était terriblement énergique et même à la datcha, où nous allions en été quand elle ne travaillait déjà plus, elle a monté perpétuellement des spectacles avec des enfants. Elle était dans son élément, bien sûr. Et elle est morte en mai 1995 - mon père en 1951 - elle avait donc presque 88 ans. Elle avait vécu tout cela et elle est morte, simplement à cause de son cœur.

Elle avait un ton un peu déclamatoire, vous savez [rire]. Parce que... voilà, par exemple, on vient me perquisitionner, ce sont quand même des tchékistes, des guébistes soviétiques, ils perquisitionnent et elle leur dit : « Je dois aller aux toilettes ». Et elle y va. Et eux lui disent : « Oui, mais laissez la porte ouverte». Et elle de leur crier dessus : « Même vos frères aînés, les satrapes de Ežov et de Berija ne se permettaient pas de me parler ainsi. » Tout ça sur un ton si théâtral et pathétique, mais qui leur faisait un peu peur. Et puis, la pauvre, elle a eu à subir mon arrestation et mon procès. Et là, même chose, le procès suit son cours, le juge parle, puis le procureur, l'avocat, et ma mère, bien sûr, qui est au premier rang, bien coiffée, belle, une vieille dame qui ressemble à Ahmatova, parce qu'elle lui ressemble, pas vrai ? Je veux dire, avec tout ce style, etc. Elle est là, au premier rang. Je dis mon dernier mot, assez modeste, et on doit déjà m'emmener. Bien sûr, tout le monde se tait, qu'est-ce que vous voulez qu'on dise encore ? Et tout à coup ma mère : « Mon fils, je suis fière de toi ! » Terrifiant ! J'en ai même un peu rougi de confusion.

Je vais vous expliquer. Ce n'est pas que ma mère manquait de goût, mais voilà... La vie soviétique était pleine d'ennui, de grisaille. Je vous ai déjà parlé du camp. Qu'est-ce qui rend le camp mauvais, affreux ? Le plus terrible, croyez-moi, ce n'est pas l'absence de femmes, comme beaucoup le pensent, ni la mauvaise alimentation. Pour moi, et pour beaucoup d'autres, d'ailleurs des gens très ordinaires, la chose la plus monstrueuse était l'absence de couleur. Le camp tout entier était monochrome, il avait la couleur de ta chemise ou de ta veste fourrée, il était gris, couleur souris, parfois noir. Si on faisait attention à son apparence, et c'était mon cas, j'avais toujours les bottes cirées, et les souliers étaient ressemelés, c'étaient des souliers hauts, que je m'étais fait faire spécialement dès ma seconde année, pas en cuir artificiel comme chez tout le monde mais des vrais, et je m'étais fait faire un costume, un costume ordinaire de détenu, mais quand même légèrement différent, et ainsi de suite. Autrement dit, si on soigne son extérieur, on peut encore garder une belle couleur noire, pas plus. Et le reste est tout gris, absolument monochrome. Donc il y a cette absence de couleur, et aussi l'absence de visages d'enfants. Et c'est très difficile à vivre, c'est une tragédie. Les femmes, il y en a. Celles qui vous donnent vos colis, une infirmière, un médecin peut-être, une institutrice à l'école du camp, etc. Il y a donc des visages de femmes, mais pas de visages d'enfants. C'est un cauchemar. Et pas de couleur. C'est pour cela que c'était si important quand mes amis m'envoyaient des reproductions de tableaux, de simples images, j'en avais pléthore, et donc c'est un thème très important de la vie au camp : la couleur et les visages d'enfants. C'est pourquoi je comprends très bien que maman ait crié cette phrase pathétique, car la vie soviétique est affreusement incolore, elle ne connaît pas de pathos. Ma mère aurait dû vivre en Italie ou en France, où tout le monde parle fort, dit de belles paroles dans une belle langue, et en y mettant les mains 
encore, et voilà, avec sa petite formule elle a mis un peu de couleur dans la banalité de ma condamnation [rire].

Donc la perestroïka, 1980... Pour moi, bien sûr, la perestroïka a commencé avant la perestroïka, c'est une jolie histoire à raconter, mais cela demanderait vingt minutes, je ne vais pas le faire.

La perestroïka. Tout évoluait très vite, tous les jours de nouveaux tabous qui sautaient. On se rencontrait entre amis et on disait : «Oh, là, là ! Tu te rends compte, ça aussi, c'est devenu possible, et ça aussi, et ça aussi. » Bien entendu, nous aussi prenions part à tout cela, pour que justement ça, et ça, et ça deviennent possibles. Mais en fait, en 86 et 87 , je reviens au travail académique, je m'occupe assez intensivement du XIX ${ }^{\mathrm{e}}$ siècle, et cela m'intéresse beaucoup. C'est mon cher vieux $\mathrm{XIX}^{\mathrm{e}}$ siècle, dont je suis formellement, officiellement spécialiste. On prépare alors la publication d'un dictionnaire remarquable, Les écrivains russes du XIX et $d u$ début $d u X X^{e}$ siècle, et ce n'est pas encore fini, je crois qu'on en est à la lettre $《 \mathrm{R} »^{7}$ [rire]. Le travail avait commencé alors que j'étais encore en camp. C'est vraiment un très beau travail qui a réuni autour de lui beaucoup d'excellentes forces scientifiques russes, historiques et littéraires. Quand même, ce sont davantage des spécialistes littéraires que des historiens, parce que les historiens, pardonnez-moi, c'est une confrérie très à part et, pour l'essentiel, assez monstrueuse. Partout, du reste, pas seulement en Russie. Avec les littéraires, c'est beaucoup plus facile, parce que, à l'époque soviétique, l'étude de la littérature remplaçait l'idéologie jusqu'à un certain point. Il ne pouvait y avoir de discussion authentique entre historiens, que ce soit au sujet des années vingt, trente ou quarante, peu importe. Mais il pouvait y avoir une discussion, même au second degré, à propos de Platonov ou Mandelstam, de Bulgakov ou Cvetaeva, on pouvait discuter des mêmes choses en parlant de Puškin, Herzen, Lomonosov... Non, pas Lomonosov, mais je dirais Puškin, Herzen, Deržavin, Fonvizin, les slavophiles, etc. Les spécialistes de littérature étaient très nombreux, et les meilleurs d'entre eux, toute une génération, se concentrèrent autour de ce dictionnaire. Quand je fus libéré, il me fallait bien travailler quelque part, gagner de l'argent, et il se trouve qu'on m'a proposé d'écrire des recensions internes sur les articles du dictionnaire. Je me suis plongé avec délice dans ce bonheur, cette joie d'écrire ces comptes rendus, parce que je pouvais rester toute la journée à la bibliothèque publique de Leningrad, lire je ne sais quelles revues des années 1810. C'était pour moi un vrai bonheur, oui. Cela me prenait énormément de temps. Je me disais même : « Seigneur, se pourrait-il que moi, qui ai perdu 17 ans à faire tout ce que j'ai fait dans les années soixante-dix — car, bien sûr, je m'occupais $\mathrm{du} \mathrm{XIX}^{\mathrm{e}}$ siècle mais aussi, parallèlement, de toute cette histoire du goulag, je recueillais des Mémoires, des documents, et j'ai quand même publié tous nos recueils—, je pourrais, moi, revenir à une profession normale, plus tranquille?»

7. Russkie pisateli. 1800-1917 : Biografičeskij slovar' [Les écrivains russes. 1800-1917. Dictionnaire biographique], M. : Sovetskaja enciklopedija, t. 1, 1989, t. 2, 1992, t. 3, 1994, t. 4 (M.-P.), 1999, t. 5 (P.-S.), 2007. (NdT) 
Mais voilà que la perestroïka arrive. Plus question de profession normale. Impossible, parce que tous les jours, il se passe quelque chose de neuf, d'intéressant. Des gens sont libérés des camps, tout doucement. Et puis commence aussi ce qu'on a appelé une « agglutination » (kučkovanie) des jeunes gens : ils s'agglutinent autour de toutes sortes d'initiatives, dans toutes les villes se forment des clubs « Perestroïka », où tout le monde discute de « ce qui est bien et de ce qui est mal $»^{8}$. Démocratie ! Moi, je n’y vais pas, ça ne m'intéresse pas. Je continue à lire Puškin et les revues du $\mathrm{XIX}^{\mathrm{e}}$ siècle [rire], tandis que ça, ça ne m'intéresse pas, je vous le jure. Mais quand même, peu à peu, cette fameuse époque contemporaine finit par percer chez moi aussi. Et voici qu'on me raconte (pour l'essentiel, je vis déjà à Moscou et non plus à Leningrad) qu'un nouveau groupe s'est créé, et qu'il s'appelle Memorial. «Et alors, qu'est-ce qu'ils veulent? " Eh bien, en fait ils veulent ce que vous, vous avez voulu. » Ce que nous avions déjà écrit autrefois, au milieu des années soixante-dix, en préface à notre recueil — c'est carrément ce programme qu'ils veulent réaliser. Pas seulement ériger un monument aux victimes (c'est simple, pour que l'État reconnaisse ces victimes), mais qu'il y ait aussi, à côté du mémorial, des archives ouvertes, un musée, une bibliothèque, une sorte de centre éternel de lutte contre cette saloperie que nous appelons souvent goulag. OK, appelons-la goulag nous aussi, pour le moment.

Bien, j'écoute tout cela, mais quand même je ne m'engage pas encore, j'écris plutôt des livres un brin plus proches de ce nouveau goût du temps. Je réunis des vieux documents, il y a là-bas [à Memorial $(\mathrm{NdT})$ ] une collection d'archives particulières, ce sont les Mémoires de paysans tolstoïens, des adeptes de Tolstoj qui avaient vécu dans diverses communautés. Ils ont connu une histoire tragique. On commença à les persécuter dès les années vingt, et, en 39, ce fut la catastrophe avec la loi sur le service militaire universel. Soit dit en passant, aucun historien ne prête attention au rôle énorme que cette loi a joué dans la conscience intellectuelle des citoyens soviétiques. Ce fut une loi très importante, et ces paysans, qui vivaient quelque part dans leurs villages, qui avaient leurs communautés, travaillaient la terre de concert et tâchaient de rester ni vus ni connus ; avant la loi, quand ils refusaient de partir à l'armée, on ne leur faisait rien, mais, à partir de 39, ils continuèrent de refuser et on commença à les envoyer en camp. Puis, ce fut 41, et comme ils continuèrent à refuser l'armée, on se mit à les fusiller les uns après les autres. Or, vous vous rappelez peut-être que les classes d'âge mobilisables, qui ont évolué pendant la guerre, allaient jusqu'à 45 ans : cela signifie que toute cette génération, celle des vrais tolstoïens, a été balayée, anéantie.

Certains tolstoïens ont quand même survécu, ils ont réapparu progressivement, surtout ceux qui avaient été arrêtés en 1939 : en 41, ils étaient déjà dans des camps et bien sûr, personne n'est allé les chercher pour les envoyer au front, c'est pourquoi ils sont restés en vie et ont fini par sortir des camps. Être enfermé dans un camp au bon moment, c'est encore un sujet très important : à quel moment c'est une chance,

8. Allusion à un poème pour enfants de Majakovksij : « Čto takoe horošo i čto takoe ploho [Qu'est-ce qui est bien et qu'est-ce qui est mal] ». (NdT) 
à quel autre une malchance. C'est un peu la même histoire que l'arrestation de mon père. Je pourrais donner beaucoup d'exemples de ce genre, à différentes périodes de l'histoire soviétique. Ces tolstoïens survivants avaient la plume très facile. Comme Lev Nikolaevič Tolstoj, ils aimaient écrire leurs Mémoires. Mémoires qu'on a commencé à collectionner chez leurs camarades, leurs collègues à Moscou, car dans les années soixante-dix il y avait encore des enfants de gens qui avaient été proches de Tolstoj et qui étaient encore bien en vie. En outre, il y avait un écrivain intéressant, Mark Popovskij qui a émigré par la suite, qui s'est intéressé aux tolstoïens et qui a écrit un livre sur eux ${ }^{9}$; il a écrit trois livres importants en samizdat, qui furent publiés par la suite à l'étranger, l'un sur l'archevêque Luc (un remarquable évêque et médecin russe, Vojno-Jaseneckij), un autre sur Vavilov et ce troisième sur les tolstoïens. Mais Popovskij a émigré, les archives des tolstö̈ens sont ici, donc je me mets d'accord avec une édition pour publier leurs Mémoires. Je prépare cette publication, j'entre en correspondance avec ceux qui sont encore en vie, et tous ces vieillards me répondent, m'envoient des photos, une masse de choses intéressantes, et les archives grossissent. Et puis on m'en confie la conservation et ce fonds devient une partie des archives de Memorial. Le fonds des « Mémoires des paysans tolstoïens ». Ce qui fait que je m'occupe quand même du $\mathrm{Xx}^{\mathrm{e}}$ siècle, bien que je n'en aie pas envie, car j'aime tant mon $\mathrm{XIX}^{\mathrm{e}}$ siècle [rire], mais je ne peux faire autrement.

C'est alors que j'apprends qu'il y a un groupe, une petite bande, qui fait des choses très bien, d'accord, mais je ne m'y intéresse pas tellement. Cependant au printemps 88 , j'apprends que ces jeunes gens organisent une rencontre déjà très pratique pour savoir comment rassembler des informations sur les victimes passées. Tant qu'ils avaient fait signer des pétitions dans la rue, réalisé de véritables exploits héroïques, ils ne m'intéressaient pas beaucoup, je vous l'assure, c'est qu'il y avait déjà un grand nombre de ces jeunes militants. Et c'est dommage qu'ils m'aient intéressé, parce que sinon, je pourrais être aujourd'hui, peut-être, un homme absolument heureux, j'enseignerais l'histoire ou la littérature russe, par exemple à la RGGU, je mènerais des séminaires, je dirais : « Tiens, j’ai un ami en France qui s'appelle Alain Blum, il a écrit un bon livre, nous en avons parlé, c'est important. » Mais j'ai commis cette terrible erreur. Bien sûr, je plaisante, ce n'était pas une erreur. Bref, quand j'ai su tout cela, j'ai réuni tout mon réseau, c'est-à-dire mes proches, mes amis, ceux qui rencontraient Memorial pour la première fois ; quelques-uns sont morts depuis. Il y avait là mon ami Aleksandr Jul'evič Daniel', alias Sanja, Sergej Adamovič Kovalev, et Larisa Iosifovna Bogoraz. Je leur dis : «On y va ». Et nous y sommes allés. C'était au printemps 88. On arrive, et on découvre de merveilleux jeunes gens qui se sont réunis et qui se demandent tout à fait sérieusement comment mettre au point un questionnaire qui pourrait être rempli aussi bien par les anciennes victimes que par leurs familles. Ce qui était loin d'être simple. Ils n'étaient pas des historiens ou des sociologues professionnels,

9. Mark Popovskij, Russkie mužiki rasskazyvajut... Posledovateli L.N. Tolstogo v Sovetskom Sojuze, 1918-1977 [Les paysans russes racontent... Les adeptes de L.N. Tolstoj en Union soviétique, 1918-1977], Londres : Overseas Publications Interchange LTD, 1983. (NdT) 
il y avait là des physiciens, des chimistes, des mathématiciens, particulièrement nombreux ceux-là. Il y avait bien aussi une brillante littéraire, mais elle était spécialiste de l'Antiquité et non de ces questions. C'était une bonne troupe, ils m'ont beaucoup plu. Je leur ai demandé des détails sur eux. J'ai appris qu'ils avaient constitué un groupe baptisé Memorial, qui s'était détaché d'un club Perestroïka au début de l'automne 87. Tandis qu'au club, on discutait de questions de politique générale, ces jeunes gens formulèrent leur idée d'un monument, puis celle d'un complexe d'activités, comprenant monument, archives, musée, bibliothèque. Et ils avaient appelé tous les volontaires, tous ceux que cela intéressait, à en parler, d'où cette première réunion. Ils étaient encore peu nombreux quand je les ai rencontrés, peut-être 15 ou 12. Mais déjà leur lettre - car ils avaient écrit une lettre au pouvoir - s'était diffusée dans toute l'Union soviétique. Et alors des groupes similaires ont commencé à se former dans différentes villes, des gens se sont mis à signer la lettre, elle était envoyée à des adresses privées, les signatures étaient rassemblées à Moscou et pour finir, il y eut cet événement célèbre, lorsqu'à la XIX ${ }^{\mathrm{e}}$ conférence du parti, en fait la dernière qu'il y ait eu, Jurij Afanas'ev, qui y était délégué, déposa devant Gorbačev toutes ces signatures, ces volumes, 50000 signatures ou plus réunies de partout. Et alors Gorbačev a dit quelque chose du genre : « Il faut, il faut ériger un monument. " Aujourd'hui, je comprends que les choses ne se sont pas passées tout à fait ainsi, que c'était beaucoup plus intéressant. Je l'ai su après, quand je suis allé — j'étais le premier à y avoir accès — aux archives du Politburo, et aussi quand nous avons préparé notre dossier pour la Cour constitutionnelle. C'est aussi moi qui ai trouvé la résolution du Politburo au sujet de Memorial, de même que celle du Comité du parti de la région de Moscou, etc. C'est seulement alors que j'ai compris qu'au Politburo les idées de Memorial étaient passées difficilement, mais à l'époque je n'en avais rien su.

Ces jeunes gens me plurent parce qu'ils s'étaient donné une tâche très concrète, mettre au point un questionnaire, le diffuser, etc. J'ai donc adhéré formellement au groupe. Sanja Daniel' ne l'a pas fait, mais moi, oui, c'est-à-dire que j'ai posé ma candidature, ils l'ont discutée et acceptée, mais j'étais déjà, peut-être, le $16^{\mathrm{e}} \mathrm{ou}$ le $17^{\mathrm{e}}$ inscrit. Je crois que nous étions 20 en tout dans ce groupe. Lena Žemkova, qui est aujourd'hui la directrice exécutive de Memorial, connaît très bien toute cette histoire, car elle a participé aux origines, elle sait comment ils se sont rencontrés au tout début, se sont séparés du club Perestroïka, ont collecté des signatures, comment ils ont persuadé je ne sais quels metteurs en scène de leur prêter des foyers de théâtre pour se réunir. Une fois même, ils ont échoué dans un commissariat, mais ont été relâchés deux heures après, bref tout un passé héroïque, une sorte de période héroïque, qui m'a précédé. Moi, je n'avais rien à voir avec cet héroïsme, mais j'aimais leur volonté de faire quelque chose. J'ai donc fréquenté les réunions de ce groupe et c'étaient vraiment des gars très sympathiques. Bien sûr, seuls certains d'entre eux recherchaient ce qui m'était proche, je veux dire l'aspect historique des choses et tous les problèmes qui en découlent. Les autres avaient quand même pour orientation intime l'avenir démocratique de notre pays, et c'est très bien, c'était magnifique, mais moi je m'intéressais à l'autre aspect. Dès le début, c'est ce que 
j'ai commencé à faire petit à petit et j'ai attiré mes amis. Sanja Daniel' m'a aidé, Nikita Ohotin m'a aidé énormément à réunir des informations de première source et beaucoup de matériaux historiques primaires furent initialement entreposés chez lui, parce qu'avec ses quatre enfants il avait eu droit à un grand appartement. En mai ou peut-être à la fin avril 1988, peu après que j'eus fait sa connaissance, Memorial tint son premier meeting, mais j'étais alors parti à Leningrad, je vivais encore entre les deux villes à cette époque. Pour autant que je sache, ce fut son premier meeting moscovite, c'était quelque part loin du centre, au métro Sokol, mais déjà on y fit parler Sergej Adamovič Kovalev. Et Kovalev y avait déjà amené Saharov.

L'étape suivante pour notre groupe fut de lancer une collecte nationale pour fonder ce centre, ce futur Memorial. Mais comment l'annoncer, nous n'avions pas de journal, le mot même de « Memorial »n'était jamais publié, même si on le connaissait « en interne ». C'est alors que nous avons eu la chance de tomber sur un homme remarquable : Jura Ščekočihin. C'était un journaliste très doué, intéressant, un petit homme vif plein de talent, par la suite il a été député pendant des années, mais il est mort ou bien on l'a assassiné, ce n'est pas clair. Javlinskij, qui en avait été très proche, est absolument certain qu'il a été sciemment empoisonné. Dima Muratov, le rédacteur en chef de Novaja gazeta est sûr de la même chose. Et voilà qu'il y eut cet entretien sensationnel entre le nouveau président Medvedev et Gorbačev ainsi que Muratov, lorsqu'il invita ces deux derniers pour leur exprimer ses condoléances à propos de l'assassinat de Markelov et de Baburina en plein centre de Moscou et qu'il s'excusa de ne pas le faire publiquement. Une rencontre étrange, mais elle a bien eu lieu. Et au cours de cet entretien, Dima demanda à Medevedev : «Pourquoi n'y a-t-il pas d'enquête sur l'affaire Ščekočihin ? Elle a été interrompue. » Et Medvedev, devant eux, a aussitôt téléphoné au chef du comité d'enquête, qui lui promit de reprendre l'investigation.

Eh bien, Ščekočihin fut le premier à publier dans la presse écrite un appel à réunir des fonds pour le futur mémorial. C'était assez important, car il était alors, je crois, journaliste à Literaturnaja gazeta [la Gazette littéraire], puis il a travaillé au ministère de la Culture. Il réussit, je ne sais comment, à faire ouvrir un compte. Mais il fallait quelqu'un pour le gérer. Alors notre groupe a eu l'idée que ce serait un conseil social (obščestvennyj sovet) de Memorial. Mais comment le créer ? On recommence à recueillir des signatures où on peut, parmi les principaux antistaliniens du pays. On en trouve 16 ou 17 et on forme ce conseil de cette façon un peu bizarre. Qui y avait-il là ? Saharov, bien sûr, était le premier. On avait sollicité aussi Solženicyn, qui se trouvait alors dans l'émigration et qui nous envoya un télégramme où il expliquait qu'il ne pouvait pas parce qu'il était loin, etc. Il y avait aussi El'cin, élu du peuple. Vous comprenez, c'était une sorte de hit-parade de l'antistalinisme au milieu de l'année 88. Saharov, Solženicyn, les écrivains Šatrov, Rybakov - l'auteur des Enfants de l'Arbat, ce roman qui avait fait tant de bruit. Evtušenko, bien sûr, incontournable. Ales' Adamovič, Bulat Okudžava. Bref, je dois dire, une constellation de gens vraiment superbes. Je crois qu'il s'est réuni en tout à deux reprises. Mon principal souvenir de Boris Nikolaevič El'cin date de ce conseil, c'est intéressant, mais c'est un peu un autre sujet, qu'il faudrait raconter. 
Donc le Conseil est créé. Mais bien sûr, pas question qu'il contrôle le compte bancaire. Le ministère nous dit : "Non, impossible, c'est un compte à la Sberbank. » Pendant ce temps, les gens envoient de l'argent, qui trois roubles, qui cent. En fait, nous ne savons pas combien il y en eut en tout, mais on raconte que pour l'époque c'était une très grosse somme. Nous voulions obtenir que le Conseil puisse contrôler ce compte. Mais le pouvoir s'y opposait de toutes les manières. Pour finir, on découvrit en 1991 qu'il n'y avait pas un seul kopeck sur le compte. Où l'argent est-il passé, qui a volé tout ça [rire], impossible de le savoir à présent. Encore heureux que l'opinion ne dise pas que c'est Memorial, que c'est nous qui l'avons volé. Encore qu'il y en a bien qui doivent le dire, mais peut-être pas, parce que nous n'avions réellement aucun accès à ce compte. Aujourd'hui, il est totalement impossible de connaître le sort de ce compte. J'ai écrit plusieurs réclamations : « Enfin, où l'argent est-il passé ? Combien y avait-il d'argent sur le compte ? » «Euh, bla-bla-bla ». Drôle d'histoire. Soit dit en passant, ce sont des durs, les employés de banque, bien pires que les guébistes, ils se couvrent les uns les autres, et ce n'est pas sur vingt ans, mais bien plus. Parce que ça peut aller trop loin. Vous comprenez, les guébistes inventaient sans cesse des petites ficelles inexistantes, des petits nœuds : on tire sur une ficelle, le nœud se défait, mais le voisin ou le supérieur n'aura rien. Tandis qu'à la banque, si on commence à tirer sur une ficelle, on ne sait pas jusqu'où ça peut aller, jusqu'à trente ans peut-être [rire].

Donc, ce conseil social avait plusieurs coprésidents : Andrej Dmitrievič Saharov, Jurij Nikolaevič Afanas'ev, Jurij Fedorovič Karjakin, Ales' Adamovič. Je ne me rappelle plus le cinquième, mais je crois bien qu'il y en avait cinq, rien que ça, comme il se doit dans un pays démocratique. Puis ils n'ont été que trois, je ne sais plus. Mais le plus intéressant dans toute cette histoire, ce fut la suite, quand il a fallu réfléchir à la façon dont on allait fonder l'association Memorial. En fait, personne n'était contre cette création. Mais, de façon générale, on est très soupçonneux en Russie, surtout dans ma génération et les générations plus âgées, quand il s'agit d'organisations formalisées. Rappelez-vous cette grande rumeur qui a couru sur la perestroïka, comme quoi elle aurait été spécialement lancée par Gorbačev et les guébistes, pour que les gens relèvent les têtes et qu'il soit plus facile de les couper ensuite. Ce fut une rumeur assez massive dans l'intelligentsia russe. Voici donc qu'arrive ce casse-tête : comment fonder l'association? Mais la décision était prise. À ce moment-là, j'étais déjà assez actif. Quoique, je vous l'assure, je n'ai jamais joué les premiers rôles. Il $\mathrm{y}$ avait des gens qui aimaient toujours aller voir des personnalités, réunir des signatures, mais moi je ne l'ai pas beaucoup fait, même si, bien sûr, il m'arrivait de discuter avec des gens importants ou d'en connaître certains. Or, quand nous avons entrepris de nous organiser en association, il s'avéra qu'il n'y avait pas de loi permettant d'en créer. Il n'y avait qu'une loi du genre de celle des « associations volontaires ». Vous savez, Spartacus, Dynamo, tous ces footballeurs. Mais quant aux associations pour la paix et autres, elles étaient créées tout simplement par décision du Comité central du parti. Donc comment faire ? Avec quelle loi ? Quels devaient être les statuts ? OK, on allait utiliser la loi des « associations volontaires ». C'est ainsi qu'est née l'idée de constituer l'Association volontaire d'éducation historique d'URSS « Memorial ». 
Cependant les associations doivent obligatoirement compter des personnes morales parmi leurs membres fondateurs. Où les prendre ? À cette époque, les plus libérales étaient les unions artistiques. Nous avons donc fait appel aux cinéastes, aux architectes, à je ne sais quels designers, à des artistes pour qu'ils fassent partie des fondateurs de Memorial. C'est ainsi que fut créé le comité d'organisation de l'association Memorial, dont je faisais aussi partie, en même temps que ces représentants des unions artistiques. C'est ce qui permit au Comité central d'essayer de peser sur tout ce processus. Nous réussîmes à tenir une conférence préparatoire en octobre 1988. Nous la voulions définitive, mais il s'avéra que c'était impossible, car nous n'avions pas tout fait comme il fallait, conformément à la loi. En réalité nous avions tout fait comme il fallait, mais tout simplement le Comité central freina la création de Memorial à travers les unions artistiques. Histoire de retarder un peu le processus. Mais les 27 et 28 janvier 89 nous réunîmes une conférence constitutive et l'association fut créée. Bien sûr, il ne pouvait y avoir de directoire, nous étions démocratiques, n'est-ce pas, alors un directoire, vous pensez ! On a donc décidé que le directoire porterait le nom de Collège de travail. Et voilà comment nous avons vécu pendant quelque huit ans avec ce Collège de travail. Du coup, personne ne voulut nous enregistrer, bien qu'il y ait eu à cette conférence des représentants de 220 villes soviétiques. 220 groupes « Memorial » dans tout le pays. Un problème follement intéressant, qu'il faudrait évoquer séparément, et longuement, ce sont bien sûr nos relations avec les Memorial d'Ukraine, de Biélorussie, des pays Baltes. C'était l'embryon de beaucoup d'évolutions qui se sont faites dans ces pays. Les uns disaient : « Nous adhérons », mais d'autres disaient : « Pour rien au monde. Nous avons notre propre tragédie, séparée, nous allons agir de notre côté. » Et ainsi de suite. Quoi qu'il en soit, nous avons créé cette organisation en janvier 1989. En décembre Andrej Dmitrievič Saharov fut élu président d'honneur et il mourut le même mois. Pendant toute cette année 89 , nous portions nos papiers partout où nous pouvions pour nous faire enregistrer, mais bien sûr, on nous renvoyait d'une instance à l'autre et personne ne voulait nous enregistrer. Il faut dire que nous ne savions pas travailler avec ce pouvoir soviétique, qui était encore bien en place. Aux obsèques de Saharov, Gorbačev s'approcha d'Elena Georgievna Bonner et lui demanda : «Que pourrais-je faire pour vous, que pourrais-je faire ?» Elle lui répondit : « Faites enregistrer Memorial ». En pleines obsèques, devant le cercueil de Saharov. « Oui, oui, oui. » Mais il fallut encore plusieurs mois et enfin, en 1990, je ne me rappelle plus quel mois, le ministère de la Justice nous délivra une attestation qui prouvait que nous étions une personne morale. Et, aussitôt, nous fîmes jouer tout notre potentiel — or il y avait alors toute une masse de gens qui se réclamaient fièrement de Memorial - et nous fûmes représentés au Parlement de Russie et à celui de l'URSS, nous devînmes très présents au parlement de Moscou, au conseil municipal aussi, et, enfin, en mobilisant toutes nos forces, nous obtînmes notre petite maison de la rue Malyj Karetnyj, qui appartenait au ministère des Constructions mécaniques moyennes, celui qui fabriquait les bombes atomiques. Le ministère venait de la remettre en état pour ses bureaux. Et nous voilà qui débarquons. « Une maison si bien refaite, on la veut! » Au ministère, on nous répond : « On vous 
la donnerait bien, on n'y tient pas, on s'en fiche, de toute façon on sent que ça va être la fin de notre ministère [rire]. Mais, disent-ils, il y a des gens de la Mairie qui la voudraient pour y ouvrir le premier night-club de Moscou. » Rappelez-vous, c'était l'année 1990 ! Je m'en souviens très bien. La mairie de Moscou, une réunion est en cours, j'écris une note à un adjoint au maire, un homme tout à fait convenable, mais il aurait sans doute signé un papier attribuant l'immeuble au night-club. J'écris : si seulement vous y ouvrez votre night-club, si vous ne nous donnez pas cette maison, nous allons rameuter, je ne sais plus, 5000 personnes et organiser une manifestation contre vous en personne [rire]. C'est comique, mais le fait est que Memorial organisait vraiment des manifestations, c'était l'époque, il y avait des dizaines de milliers de manifestants... Bref nous avons eu cette petite maison, nous y vivons toujours, et, pour l'instant, on ne nous a pas encore fichus dehors.

Traduit du russe par Wladimir Berelowitch 